\title{
Evaluación del índice de dificultad de las preguntas de la prueba general de admisión 2014-II a la Universidad Nacional Mayor de San Marcos. El caso de la asociaciones lineales de un agrupamiento de carreras profesionales
}

Evaluation of the difficulty index of questions from the general admission test 2014-II

at San Marcos National University. The case of a linear associations grouping careers

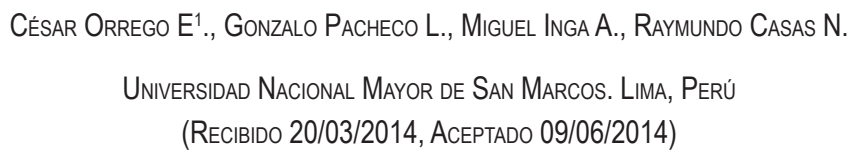

\begin{abstract}
RESUMEN
El presente trabajo descriptivo-correlacional afirma lo siguiente: primero, las preguntas elaboradas, comunes a los postulantes a Humanidades, Ciencias Sociales y EconómicoEmpresariales, de la Prueba General de Admisión 2014-II varían en la escala extremadamente difícil-extremadamente fácil. En general, la mayoría de las preguntas califican como difícil, intermedio y fácil. Sin embargo, existe un patrón sistemático a la mayoría de las áreas académicas: por los resultados, los ítems relativos al área curricular Matemática y Ciencia, Tecnología y Ambiente califican como extremadamente difícil y Habilidad LógicoMatemática, como extremadamente fácil. Segundo, a pesar de que los coeficientes de correlación son elevados, positivos y estadísticamente significativos, entre las carreras profesionales de la ACP analizadas, existiría la posibilidad de dos perfiles de postulantes: por un lado, Humanidades y Ciencias Sociales y, por el otro lado, Económico-Empresariales. Las formas de las nubes de puntos, entre los pares de carreras profesionales, permitirían así formular conjeturas razonables.
\end{abstract}

Palabras clave: examen de selección, índice de dificultad, Perú, Universidad Nacional Mayor de San Marcos

\section{ABSTRACT}

This descriptive corelational work states the following: First, the questions customary to applicants for the Humanities, Social Sciencies and Economics of Business General Admission Test 2014 - II vary in scale from Extremely Easy. In general, most of the questions qualify as Hard, Medium and Easy. 
EVALUACIÓN DEL ÍNDICE DE DIFICULTAD DE LAS PREGUNTAS DE LA PRUEBA GENERAL DE ADMISIÓN 2014-II

However, there is a systematic pattern in most academic areas: According to the results, items related to the curricular area Math and Science, Technology and Enviroment qualify as Extremely Difficult, while the Mathematical Logical Ability qualify as Extremly Easy. Second, despite the fact that the correlation coefficient is high, positive and statistically significant among the analyzed careers (cc), it is possible for two applicant profiles to exist: First, Humanities and social Sciences and Second, Economics of Bussiness. The shapes of the clouds of points between the pairs of professional careers, would allow for these reasonable assumptions.

Keywords: Selection exmination, index of difficulty, Peru, National University of San Marcos

\section{INTRODUCCIÓN}

El presente informe, puesto a disposición de la comunidad académica, presenta los resultados de un trabajo de investigación de tipo descriptivo-correlacional, relativo al índice de dificultad de las preguntas de la Prueba General de Admisión 2014-II (ejecutada en el mes de marzo de 2014) en la Universidad Nacional Mayor de San Marcos, de un agrupamiento de carreras profesionales.

El agrupamiento de carreras profesionales (ACP) es un concepto unificador de elementos, en este caso opciones profesionales, enlazados mediante el constructo destreza cognitiva, teniendo en cuenta que los postulantes tendrían, al estar agrupados, igual o similar nivel de destreza cognitiva. En esta oportunidad, el agrupamiento de carreras profesionales corresponde a Humanidades, Ciencias Sociales y Económico-Empresariales. Por supuesto, al interior de cada carrera existe un número determinado de escuelas académicas.

Los objetivos del trabajo son, primero, calcular el índice de dificultad de cada pregunta contestada por los postulantes de cada una de las carreras profesionales del ACP y, segundo, estimar el coeficiente de correlación de cada par de carrera profesional involucrada en el respectivo ACP.

Para cumplir los objetivos, el documento está dividido en seis secciones. La primera cubre los aspectos generales (normas establecidas) del examen. A continuación, la segunda sección presenta las preguntas y las hipótesis de investigación. La siguiente sección presenta lo relativo al índice de dificultad, lo cual implica la fórmula de cálculo y los intervalos de calificación de la pregunta. La cuarta sección está dedicada al tratamiento de los datos, por ello se titula Método. La penúltima sección trata sobre la presentación de los resultados. La última parte está dedicada a presentar las conclusiones.

Las tablas y figuras estadísticas han sido desarrollados empleando los soportes lógicos computacionales SPSS y Excel, teniendo como fuente las bases de datos solicitadas a la Unidad de Informática de la Oficina Central de Admisión por la Oficina de Estudios y Elaboración de Pruebas. 


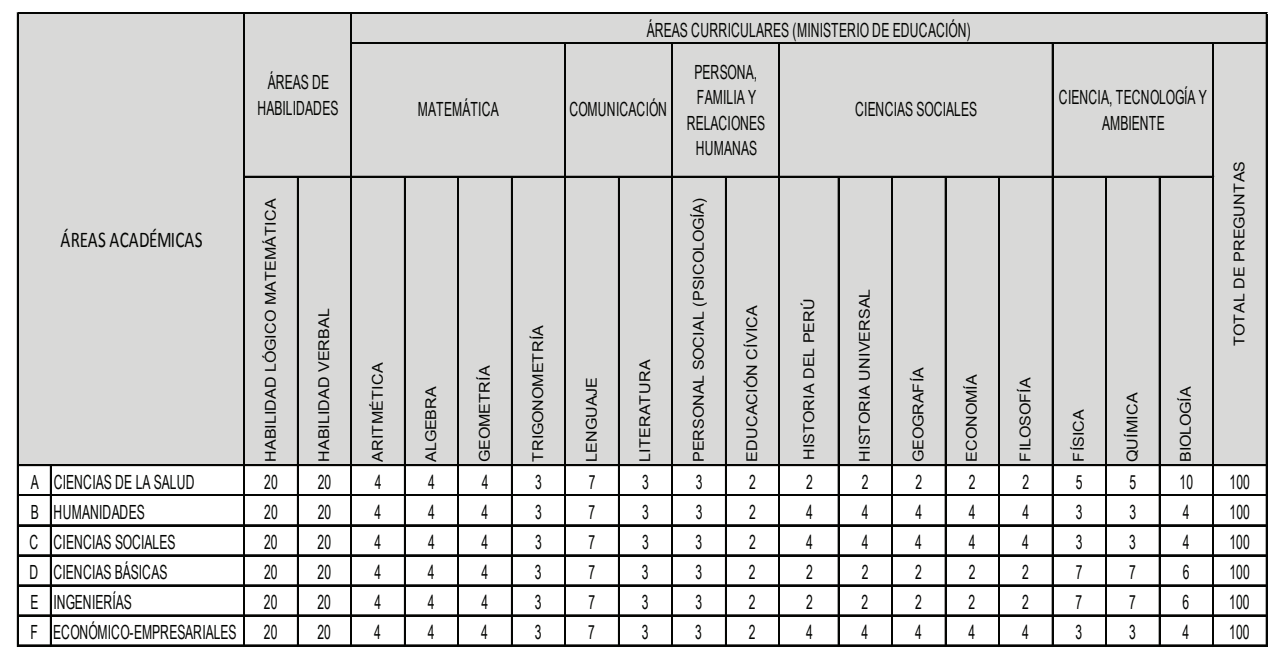

\section{ASPECTOS GENERALES}

El examen de admisión (EA) a la Universidad Nacional Mayor de San Marcos (UNMSM) es una prueba de 100 preguntas de tipo objetivo que los postulantes al ACP intentarán resolver en un lapso de 3 horas. Las preguntas son de selección múltiple, con cinco alternativas de respuesta: una de ellas es la correcta y el resto de preguntas son distractores.

La prueba, según el reglamento de admisión (UNMSM, 2013), comprende ítems agrupados en dos partes: 40 preguntas de habilidades (lógico-matemática y verbal, cada una con igual número de reactivos), comunes para todas las áreas académicas; y 60 de conocimientos (áreas curriculares definidas por el Ministerio de Educación), los cuales varían, numéricamente, según el área académica (véase tabla 1).

Para cubrir una vacante determinada por la Escuela Académico Profesional (EAP), el postulante deberá cumplir dos condiciones (UNMSM, 2013): obtener un puntaje igual o mayor a 800 puntos (salvo las EAP de Educación Física y Danza) y ocupar una vacante de acuerdo al orden de mérito.

La primera condición, es decir, el puntaje total de la prueba que logra el postulante, se obtiene a partir de la siguiente suma: pregunta correcta, 20 puntos; pregunta incorrecta, menos 1.725 puntos; y pregunta no respondida o más de una marca, 0 puntos.

La aplicación del examen se lleva a cabo en la ciudad universitaria de la UNMSM. El ACP es para fines del momento evaluativo de la siguiente manera: Ciencias de la Salud (A), Ciencias Básicas (D) e Ingenierías (E) forman un conjunto; y Humanidades (B), Ciencias Sociales (C) y Económico-Empresariales (F), otro conjunto. Durante el proceso de admisión 2014-II se establecieron los días sábado 8 y domingo 9 de marzo como fechas evaluativas del primer y segundo grupo, respectivamente. Finalmente, la calificación de la prueba es, entre otras características, anónima y automatizada. 
Evaluación del ÍNDICE dE DIFICULTAD DE LAS PREGUNTAS DE LA PRUEBA GENERAL DE ADMIIIÓN 2014-॥

\section{PROBLEMA}

A fin de llevar adelante el trabajo, se formularon dos preguntas de investigación. La primera está enunciada en los siguientes términos: ¿cuál es el índice de dificultad de cada pregunta de la Prueba General de Admisión 2014-II del ACP conformado por Humanidades, Ciencias Sociales y Económico-Empresariales?

La segunda pregunta es: ¿cuál es el grado de asociación lineal entre los índices de dificultad de las preguntas del examen de admisión 2014-II a la UNMSM de Humanidades, Ciencias Sociales y Económico Empresariales?

A partir del segundo problema de investigación, las hipótesis derivadas son las siguientes:

Hipótesis 1

Los índices de dificultad de las preguntas del examen de admisión 2014-II a la UNMSM de Humanidades y Ciencias Sociales están asociados, linealmente, de forma directa.

\section{Hipótesis 2}

Los índices de dificultad de las preguntas del examen de admisión 2014-II a la UNMSM de Humanidades y Económico-Empresariales están asociados, linealmente, de forma directa.

\section{Hipótesis 3}

Los índices de dificultad de las preguntas del examen de admisión 2014-II a la UNMSM de Ciencias Sociales y Económico-Empresariales están asociados, linealmente, de forma directa.

\section{Índice de dificultad}

El índice de dificultad (ID) de la pregunta (j), según el ACP (g), se define en los siguientes términos.

$$
I D_{j}^{g}=\frac{P R_{1}^{g}}{P R_{0}^{g}+P R_{1}^{g}}
$$

Donde:

$$
\begin{aligned}
& \text { PR0 = Pregunta contestada incorrectamente } \\
& \text { PR1 }
\end{aligned}
$$

En otras palabras, el índice expresa el número respuestas correctamente contestadas de una pregunta respecto del número total de respuestas (correcta e incorrectamente contestadas) de la pregunta. Ahora bien, el índice de dificultad, que cae entre los límite 0 y 1 , califica a la pregunta de acuerdo al valor y su ubicación en una escala de intervalo desarrollado. Los intervalos de calificación y clasificación de la pregunta son los siguientes: 


\begin{tabular}{cc}
\hline Valor & Clasificación \\
\hline$\leq 0.2499999$ & Extremadamente difícil \\
$0.25-0.449999$ & Difícil \\
$0.45-0.549999$ & Intermedio \\
$0.55-0.749999$ & Fácil \\
$\geq 0.75$ & Extremadamente fácil \\
\hline
\end{tabular}

De acuerdo al valor del índice, la pregunta califica en los siguientes intervalos: Extremadamente difícil a extremadamente fácil.

\section{MÉTODO}

Los datos empleados son de fuente primaria, procesados, una vez culminado el examen de admisión, por la UI de la OCA, UNMSM.

El tratamiento de los datos ha seguido la siguiente ruta de trabajo:

\section{i. Matrices R1 ${ }^{\text {g }}$}

Los datos recuperados de la ficha óptica de la Sección de Respuestas scn los insumos básicos $\mathrm{y}$, una vez procesado por la UI, determinan las matrices ${ }^{\mathrm{g}}$. Por supuesto, los datos corresponden al examen de admisión que, posterior al tratamiento estadístico, servirán como insumo de la fórmula del índice de dificultad. Por otro lado, el número de matrices será igual a la cantidad de $\mathrm{ACP}(\mathrm{g})$ definidas en el estudio (Humanidades, Ciencias Sociales y Económico-Empresariales).

Esquemáticamente,

matriz R1 $1^{\mathrm{g}}=\left[\mathrm{a}_{\mathrm{ij}}\right], \mathrm{m} \mathrm{x} \mathrm{n}$

Sujeto los elementos (aij) a las siguientes calificaciones de las respuestas:

$\mathrm{a}_{\mathrm{ij}}\left\{\begin{array}{l}0 \text { Incorrecta } \\ 1 \text { Correcta } \\ 2 \text { Más de una } \\ \text { En blanco }\end{array} \quad(\mathrm{i}=1,2, \ldots, \mathrm{m} ; \mathrm{j}=1,2, \ldots, \mathrm{n})\right.$

Donde:

$\mathrm{i}=$ m-ésimo postulante.

$\mathrm{j}=$ n-ésima pregunta de la prueba.

Matrices ${ }^{\mathrm{R}}{ }^{\mathrm{g}}$

En el segundo paso, las matrices ${ }^{\mathrm{R}}{ }^{\mathrm{g}}$ deberán ser modificadas. Los cambios a ejecutar están explicados por el uso de la fórmula del índice de dificultad. Esto implica, esquemáticamente, lo siguiente:

matriz R2 ${ }^{\mathrm{g}}=\left[\mathrm{b}_{\mathrm{ij}}\right], \mathrm{mxn}$ 
En esta oportunidad, los elementos (bij) están sujetos a las siguientes calificaciones:

$b_{i j}\left\{\begin{array}{l}0 \text { si } a_{i j} 0 \\ 1 \text { si } a_{i j} 1 \\ 0 \text { si } a_{i j} 2 \\ 0 \text { si } a_{i j}\end{array} \quad(i=1,2, \ldots, m ; j=1,2, \ldots, n)\right.$

Donde:

$\mathrm{i}=$ m-ésimo postulante.

$\mathrm{j}=$ n-ésima pregunta de la prueba.

Por tanto, las matrices $\mathrm{R}^{\mathrm{g}}$ son, finalmente, los datos expeditos para la aplicación de la correspondiente fórmula y posterior análisis.

\section{Índice de dificultad}

El cálculo del índice de dificultad (ID) de la pregunta (i), según el ACP (g), implica trabajar con las matrices $\mathrm{R}^{\mathrm{g}}{ }^{\mathrm{a}}$. Formalmente,

\begin{tabular}{rl|}
\hline \multicolumn{1}{|c|}{${\text { Si } \mathrm{a}_{\mathrm{ij}}=0:}^{\mathrm{S}} \mathrm{Si}_{\mathrm{ij}}=1:$} \\
$\mathrm{PR}_{0}^{\mathrm{g}}=\sum_{\mathrm{i}=1}^{\mathrm{m}} \mathrm{a}_{\mathrm{i} 1}$ & $\mathrm{PR}_{1}^{\mathrm{g}}=\sum_{\mathrm{i}=1}^{\mathrm{m}} \mathrm{a}_{\mathrm{i} 1}$ \\
$\mathrm{PR}_{0}^{\mathrm{g}}=\sum_{\mathrm{i}=1}^{\mathrm{m}} \mathrm{a}_{\mathrm{i} 2}$ & $\mathrm{PR}_{1}^{\mathrm{g}}=\sum_{\mathrm{i}=1}^{\mathrm{m}} \mathrm{a}_{\mathrm{i} 2}$ \\
$\mathrm{PR}_{0}^{\mathrm{g}}=\sum_{\mathrm{i}=1}^{\mathrm{m}} \mathrm{a}_{\mathrm{in}}$ & $\mathrm{PR}_{1}^{\mathrm{g}}=\sum_{\mathrm{i}=1}^{\mathrm{m}} \mathrm{a}_{\mathrm{in}}$
\end{tabular}

\section{Diagrama de dispersión}

El penúltimo paso implica ubicar en el plano cartesiano los índices de dificultad de las preguntas según combinaciones de opciones profesionales: Humanidades (H), Ciencias Sociales (C) y Económico-Empresariales (E). Así, el diagrama de dispersión brindará información, mediante la ubicación de los puntos en el plano, respecto de algún patrón sistemático.

\section{Asociación lineal}

El último paso implica calcular el coeficiente de correlación (r) a cada una de las combinaciones - de dos-de opciones profesionales: Humanidades $(\mathrm{H})$, Ciencias 
Sociales (C) y Económico-Empresariales (E), y, posteriormente, someter las tres hipótesis a la prueba de significancia. Formalmente,

$\mathrm{H}_{0}: \mathrm{r}_{\mathrm{HC}}=0$

$\mathrm{H}_{0}: \mathrm{r}_{\mathrm{HE}}=0$

$\mathrm{H}_{0}: \mathrm{r}_{\mathrm{CE}}=0$

Lo que implica, en otros términos, independencia entre las variables. El nivel de significancia es $\mathrm{p}<0.05$ [bilateral].

\section{RESULTADOS}

El análisis de la dificultad de la pregunta se inicia observando la figura 1. Según el gráfico, se presenta tres paneles: A presenta información del índice de dificultad por pregunta de Humanidades; B, Ciencias Sociales; y C, Económico-Empresariales.

FIGURA 1

Prueba de Admisión a la UNMSM 2014-II: Índice de dificultad de las preguntas (Fecha de examen: 9 de marzo de 2014)

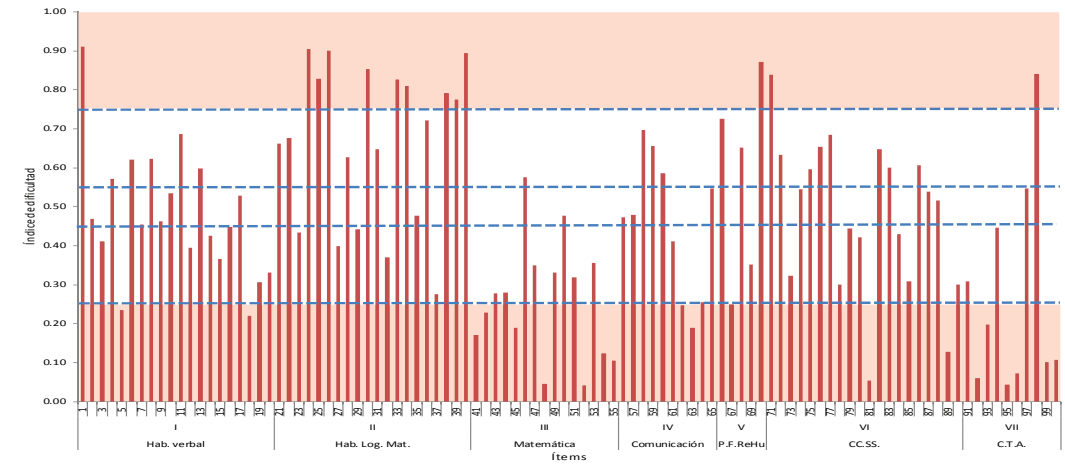

[A] B - Humanidades

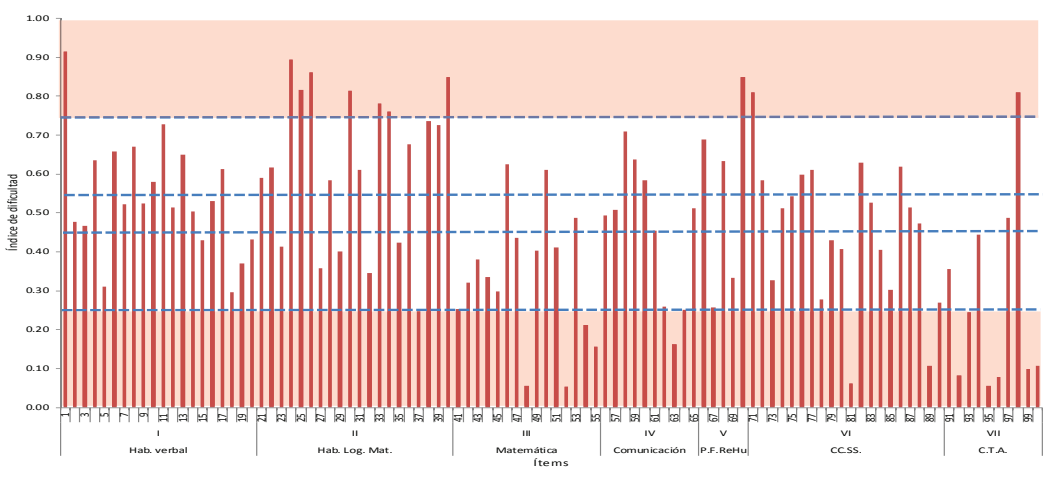

[B] C - Ciencias Sociales 
EVALUACIÓN DEL ÍNDICE DE DIFICULTAD DE LAS PREGUNTAS DE LA PRUEBA GENERAL DE ADMISIÓN 2014-॥

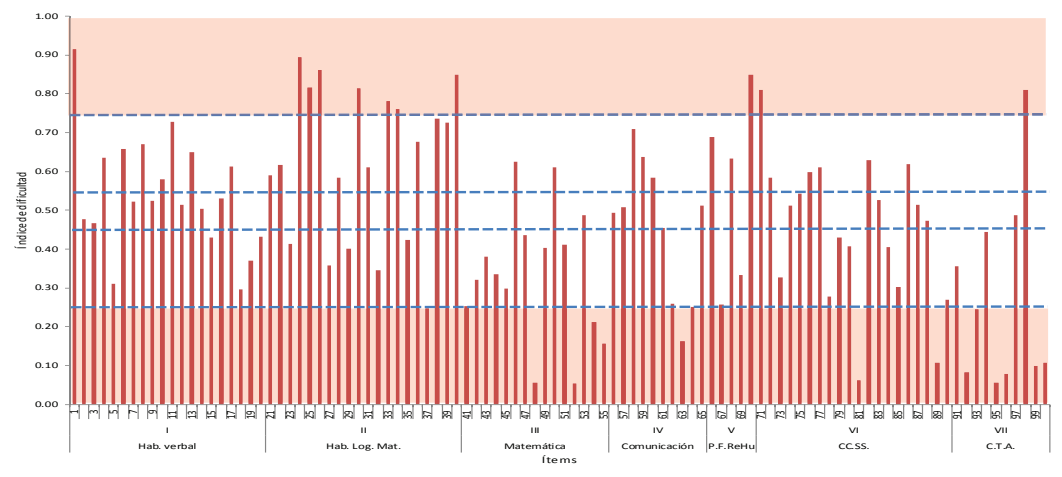

[C] F - Económico-Empresariales

La nomenclatura de la figura de barra es la siguiente: eje horizontal, I - Habilidad Lógico Matemática; II - Habilidad Verbal; III - Matemática; IV - Comunicación; V - Persona, Familia y Relaciones Humanas; VI - Ciencias Sociales; y VII Ciencia, Tecnología y Ambiente.

En general, la dificultad-facilidad de las preguntas está dentro de las líneas establecidas. La mayor cantidad de preguntas está clasificada como difícil (0.25 a 0.44999 puntos), intermedia (0.45 a 0.54999 puntos) y fácil (0.55 a 0.74999$)$. Sin embargo, la misma figura, en sus dos paneles ([A] y [B]), muestra un patrón sistemático: en el caso del área curricular Matemática, casi la mayoría de las preguntas son extremadamente difícil (0.00 a 0.24999). No es el caso, en este punto, de Económico-Empresariales (véase panel [A]). En cambio, la mayoría de las preguntas relativas al área curricular Ciencia, Tecnología y Ambiente son clasificadas como extremadamente difícil por el conjunto del ACP. Más aún, también un número importante de preguntas (9) de Habilidades Matemáticas califican, esta vez, como extremadamente fácil.

\section{DISCUSIÓN}

Una lectura rápida de lo anotado, y otros aspectos más, pueden ser apreciados en la sección Apéndice, cuadro 1A. El cuadro estadístico reúne toda la información, prestando atención a las áreas oscurecidas.

Observe, ahora, el gráfico 2. Como el gráfico anterior, presenta información en tres paneles, pero referido al valor del índice de dificultad de cada una de las preguntas, según el área profesional, agrupadas de a dos; en otras palabras, se trata de un gráfico de dispersión. Por lo tanto, la nube de puntos corresponde a las preguntas y los valores obtenidos según el área profesional. Además, la línea diagonal es equidistante a los valores de cada eje, lo cual implica valores iguales del índice de dificultad.

¿Qué información podemos extraer del gráfico? En general, en el panel [a], Humanidades - Ciencias Sociales, la nube de puntos está muy próxima a la diagonal. 
De existir una ligera desviación de la misma, la inclinación es hacia a la derecha, es decir, a Ciencias Sociales, lo que significa un nivel casi similar de índice de dificultad de la pregunta. En cambio, los paneles [b], Humanidades - EconómicoEmpresariales, y [c], Ciencias Sociales - Económico-Empresariales, revelan que sus respectivas nubes de puntos presentan patrones espaciales diferentes a lo observado en el panel [a].

En los paneles [b] y [c], la nube de puntos presenta dos características: primero, en los extremos, los puntos están ubicados muy próximos a la línea diagonal; y, segundo, entre los puntos extremos, la mayor cantidad de puntos de la nube se ubica hacia la derecha de la línea equidistante. En otros términos, los índices de dificultad de un grupo de preguntas de Económico-Empresariales se inclinan, ligeramente, hacia la clasificación intermedia y fácil.

Al parecer, los perfiles de los postulantes serían, según lo analizado, dos: por un lado, Humanidades y Ciencias Sociales; y, por el otro lado, Económico-Empresariales.

FIGURA 2

Prueba de Admisión 2014-II a Humanidades, Ciencias Sociales y EconómicoEmpresariales de la UNMSM: Diagrama de dispersión de los índices de dificultad

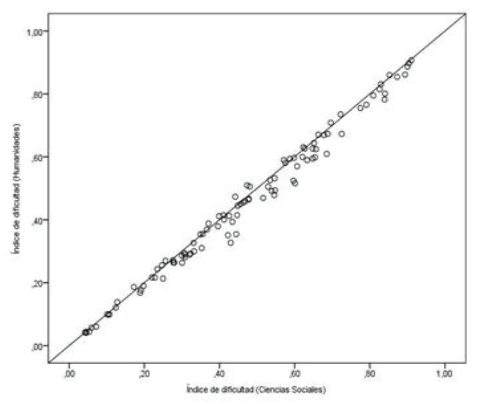

[a] Humanidades - Ciencias Sociales

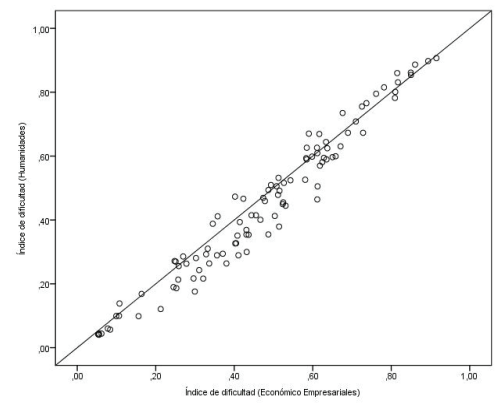

[b] Humanidades - EconómicoEmpresariales

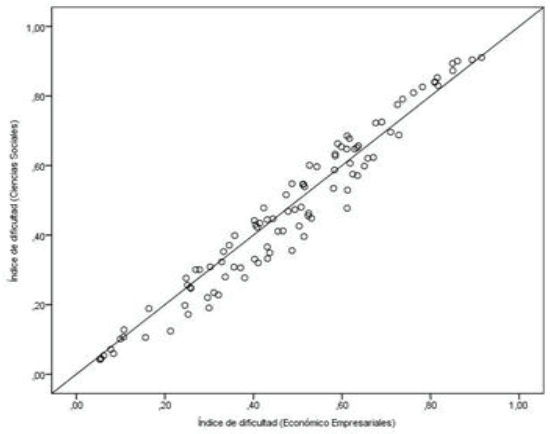

[c] Ciencias Sociales - EconómicoEmpresariales 
Analicemos, a partir de ahora, la tabla 3. La información estadística, relativa a la matriz de correlación de los índices de dificultad de las preguntas contestadas por los postulantes a Humanidades, Ciencias Sociales y Económico-Empresariales, revela la asociación lineal entre los valores de los índices.

TABLA 3

Prueba de Admisión 2014-II a Humanidades, Ciencias Sociales y Económico-

Empresariales de la UNMSM: Matriz de correlación de los índices de dificultad

\begin{tabular}{clrrr}
\hline & Humanidades & $\begin{array}{c}\text { Ciencias } \\
\text { Sociales }\end{array}$ & $\begin{array}{c}\text { Económico } \\
\text { Empresariales }\end{array}$ \\
\hline \multirow{2}{*}{ Humanidades } & Correlación de Pearson & 1 &, $994^{* *}$ &, $977^{* *}$ \\
& Sig. (bilateral) & & .000 & .000 \\
& $N$ & 100 & 100 & 100 \\
Ciencias & Correlación de Pearson &, $994^{* *}$ & 1 &, $976^{* *}$ \\
Sociales & Sig. (bilateral) & .000 & & .000 \\
& $\mathrm{~N}$ & 100 & 100 & 100 \\
Económico & Correlación de Pearson &, $977^{* *}$ &, $976^{* *}$ & 1 \\
Empresariales & Sig. (bilateral) & .000 & .000 & \\
& $\mathrm{~N}$ & 100 & 100 & 100 \\
\hline
\end{tabular}

**. La correlación es significativa al nivel 0,01 (bilateral).

Según los resultados, las asociaciones lineales directas y próximas a uno son estadísticamente significativas. Por tanto, se pueden rechazar las tres hipótesis nulas y afirmar que los valores de los índices de dificultad son muy parecidos.

\section{CONCLUSIONES}

Del análisis realizado, las conclusiones del estudio son las siguientes:

1. Las preguntas elaboradas, comunes a los postulantes a Humanidades, Ciencias Sociales y EconómicoEmpresariales, varían en la escala extremadamente difícil-extremadamente fácil. En general, la mayoría de las preguntas califican como difícil, intermedio y fácil. Sin embargo, existe un patrón sistemático a la mayoría de las áreas académicas: por los resultados, los ítems relativos al área curricular Matemática y Ciencia, Tecnología y Ambiente califican como extremadamente difícil y Habilidad Lógico-Matemática, como extremadamente fácil.

2. A pesar de que los coeficientes de correlación son elevados, positivos y estadísticamente significativos, entre las carreras profesionales del ACP analizadas, existiría la posibilidad de dos perfiles de postulantes: por un lado, Humanidades y Ciencias Sociales y, por el otro lado, EconómicoEmpresariales. Las formas de las nubes de puntos, entre los pares de carreras profesionales permitirían, así, formular conjeturas razonables. 


\section{REFERENCIAS BIBLIOGRÁFICAS}

Aiken, L. (2003). Tests psicológicos y evaluación (undécima edición). México: Pearson educación.

Landauro, C. (2012). Fundamentos teóricos del análisis clásico de pruebas (Informe $\mathrm{N}^{\mathrm{o}}$ 1). Lima: UNMSM, AID-OEEP-OCA.

Landauro, C., Inga, M. Casas, R., Miljanovich, M., Bernui, J. (2013). Diagnóstico de la prueba de admisión a la Universidad Nacional Mayor de San Marco: Proceso 2013-II (Informe $\mathrm{N}^{\circ}$ 5). Lima: UNMSM, AID-OEEP-OCA.

Universidad Nacional Mayor de San Marcos - UNMSM (2013). Reglamento de admisión 2014-II. Lima: UNMSM, Oficina Central de Admisión.

\section{ANEXOS}

TABLA $1 \mathrm{~A}$

Número de ítems según índice de dificultad de todas las áreas académicas, 2014-II

\begin{tabular}{|c|c|c|c|c|c|c|c|}
\hline \multirow{2}{*}{\multicolumn{2}{|c|}{ Área académica }} & \multicolumn{5}{|c|}{ Índice de dificultad } & \multirow[b]{2}{*}{ Total } \\
\hline & & $\begin{array}{c}<0.25 \\
\text { Ex. dificil }\end{array}$ & $\begin{array}{c}0.25-0.44999 \\
\text { Dificil } \\
\end{array}$ & $\begin{array}{c}0.45-0.54999 \\
\text { Intermedia }\end{array}$ & $\begin{array}{c}0.55-0.74999 \\
\text { Fácil } \\
\end{array}$ & $\begin{array}{l}\geq 0.75 \\
\text { Ex. fácil }\end{array}$ & \\
\hline \multirow{9}{*}{ B. Humanidades } & ICTA & $G$ & 2 & 1 & 0 & 1 & 10 \\
\hline & & 0 & 2 & 1 & 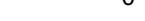 & 1 & 10 \\
\hline & CC.SS. & 2 & 7 & 5 & 5 & 1 & 20 \\
\hline & Comunicación & 1 & 3 & 3 & 3 & 0 & 10 \\
\hline & Hab. Log. Mat. & 0 & 4 & 2 & 5 & 9 & 20 \\
\hline & Hab. verbal & 2 & 8 & 4 & 5 & 1 & 20 \\
\hline & Matemática & 7 & 6 & 1 & 1 & 0 & 15 \\
\hline & P.F.ReHu & 1 & 1 & 0 & 2 & 1 & 5 \\
\hline & Total & 19 & 31 & 16 & 21 & 13 & 100 \\
\hline \multirow{8}{*}{$\begin{array}{l}\text { C. Ciencias } \\
\text { Sociales }\end{array}$} & C.T.A. & 6 & 2 & 1 & 0 & 1 & 10 \\
\hline & CC.ss. & 2 & 7 & 3 & 7 & 1 & 20 \\
\hline & Comunicación & 2 & 2 & 3 & 3 & 0 & 10 \\
\hline & Hab. Log. Mat. & 0 & 5 & 1 & 5 & 9 & 20 \\
\hline & Hab. verbal & 2 & 7 & 5 & 5 & 1 & 20 \\
\hline & Matemática & 7 & 6 & 1 & 1 & 0 & 15 \\
\hline & P.F.ReHu & 1 & 1 & 0 & 2 & 1 & 5 \\
\hline & Total & 20 & 30 & 14 & 23 & 13 & 100 \\
\hline \multirow{8}{*}{$\begin{array}{l}\text { F. Económico- } \\
\text { Empresariales }\end{array}$} & C.T.A. & 6 & 2 & 1 & 0 & 1 & 10 \\
\hline & CC.ss. & 2 & 7 & 5 & 5 & 1 & 20 \\
\hline & Comunicación & 1 & 2 & 4 & 3 & 0 & 10 \\
\hline & Hab. Log. Mat. & 1 & 5 & 0 & 7 & 7 & 20 \\
\hline & Hab. verbal & 0 & 5 & 7 & 7 & 1 & 20 \\
\hline & Matemática & 4 & 8 & 1 & 2 & 0 & 15 \\
\hline & P.F.ReHu & 0 & 2 & 0 & 2 & 1 & 5 \\
\hline & Total & 14 & 31 & 18 & 26 & 11 & 100 \\
\hline
\end{tabular}

